\title{
SOME NEW INTEGRAL INEQUALITIES ON TIME SCALES
}

\section{YUANGONG SUN}

Abstract. By introducing two adjusting parameters, we investigate some new nonlinear integral inequalities on time scales, which provide explicit bounds on unknown functions, and can be used as tools in the qualitative theory of certain classes of dynamic equations on time scales. Mathematics subject classification (2010): 26D15, 26 E70.

Keywords and phrases: Integral inequality, time scale, adjusting parameters.

\section{REFERENCES}

[1] S. HILGER, Analysis on measure chains-A unified approach to continuous and discrete calculus, Results Math. 18 (1990), 18-56.

[2] R. Agarwal, M. Bohner, A. Peterson, Inequalities on time scales: a survey, Math. Inequal. Appl. 4 (2001), 535-557.

[3] E. Akin-Bohner, M. Bohner, F. Akin, Pachpatte inequalities on time scales, Journal of Inequalities in Pure Applied Mathematics 6 (2005), article 6, 23 pages.

[4] W. N. LI, Some new dynamic inequalities on time scales, J. Math. Anal. Appl. 319 (2006), 802-814.

[5] W. N. LI, Some Pachpatte type inequalities on time scales, Computers and Mathematics with Applications 57 (2009), 275-282.

[6] W. N. LI, W. SHENG, Some nonlinear integral inequalities on time scales, Journal of Inequalities and Applications (2007), 15 pages.

[7] W. N. LI, W. Sheng, Some nonlinear dynamic inequalities on time scales, Proc. Indian Acad. Sci. Math. Sci. 117 (2007), 545-554.

[8] W. N. LI, Some delay integral inequalities on time scales, Computers and Mathematics with Applications 59 (2010), 1929-1936.

[9] D. R. ANDERSON, Nonlinear dynamic integral inequalities in two independent variables on time scale pairs, Advances in Dynamical Systems and Applications 3 (2008), 1-13.

[10] D. R. ANDERSON, Dynamic double integral inequalities in two independent variables on time scales, J. Math. Inequal., 2 (2008), 163-184.

[11] D. B. PACHPATTE, Explicit estimates on integral inequalities with time scale, Journal of Inequalities in Pure Applied Mathematics 7 (2006), article 143, 8 pages.

[12] F. H. Wong, C. C. YeH, C.H. Hong, Gronwall inequalities on time scales, Math. Inequal. Appl., 9 (2006), 75-86.

[13] F. WonG, C. C. YEH, S. L YU, C. H. HonG, Young's inequality and related results on time scales, Appl. Math. Lett. 18 (2005), 983-988.

[14] B. G. PAChPATTE, On some new inequalities related to a certain inequality arising in the theory of differential equations, J. Math. Anal. Appl. 251 (2000), 736-751.

[15] M. Bohner, A. Peterson, Dynamic Equations on Time Scales, An Introduction with Applications, Birkhäuser, Boston, 2001.

[16] M. Bohner, A. Peterson (Eds.), Advances in Dynamic Equations on Time Scales, Birkhäuser, Boston, 2003.

[17] F. W. Meng, D. Ji, On some new nonlinear discrete inequalities and their applications, J. Comput. Appl. Math. 208 (2007), 425-433.

[18] F. W. Meng, W. N. LI, On some new nonlinear discrete inequalities and their applications, J. Comput. Appl. Math. 158 (2003), 407-417. 